\title{
Electron-LO-phonon Intrasubband Scattering Rates in a Hollow Cylinder Under the Influence of a Uniform Axial Applied Magnetic Field
}

Monkami Masale

University of Botswana

Moletlanyi Tshipa ( $\square$ tshipam@ub.ac.bw)

University of Botswana https://orcid.org/0000-0001-6894-7637

\section{Research Article}

Keywords: Electron-phonon interaction , Scattering rates , Hollow cylinder , Axial magnetic field

Posted Date: July 22nd, 2021

DOI: https://doi.org/10.21203/rs.3.rs-722087/v1

License: (c) (i) This work is licensed under a Creative Commons Attribution 4.0 International License.

Read Full License

Version of Record: A version of this preprint was published at Optical and Quantum Electronics on November 6th, 2021. See the published version at https://doi.org/10.1007/s11082-021-03208-z. 


\title{
Electron-LO-phonon intrasubband scattering rates in a hollow cylinder under the influence of a uniform axial applied magnetic field
}

\author{
Do you have a subtitle? \\ If so, write it here \\ Monkami Masale • Moletlanyi Tshipa
}

Received: date / Accepted: date

\begin{abstract}
Scattering rates arising from the interactions of electrons with bulk longitudinal optical (LO) phonon modes in a hollow cylinder are calculated as functions of the inner radius and the uniform axial applied magnetic field. Now, the specific nature of electron-phonon interactions mainly depends on the character of the energy spectrum of electrons. As is well known, in cylindrical quantum wires, the application of a parallel magnetic field lifts the double degeneracy of the non-zero azimuthal quantum number states; $m \neq 0$; irrespective of all electron's radial quantum number $l$ states. In fact, this Zeeman splitting is such that the $m<0$ electron's energy subbands initially decrease with the increase of the parallel applied magnetic field. In a solid cylinder, the lowest-order; $\{l=1 ; m=0\}$ subband is always the ground state. In a hollow cylinder, however, as the axial applied magnetic field is increased, the electron's energy subbands take turns at becoming the ground state; following the sequence $\{m=0,-1,-2 \ldots-N\}$ of azimuthal quantum numbers. Furthermore, in a hollow cylinder, in general, the electron's energy separations between any two subbands are less than the LO phonon energy except for exceptionally high magnetic fields, and some highest-order quantum number states. In view of this, the discussion of the energy relaxation here is focused mainly on intrasubband scattering of electrons and only within the lowestorder $\{l=1 ; m=0\}$ electron's energy subband. The intrasubband scattering
\end{abstract}

\author{
M. Masale \\ University of Botswana, Private Bag 0022, Gaborone, Botswana \\ Tel.: +267-355-2140 \\ Fax: +267-3185097 \\ E-mail: masalem@ub.ac.bw \\ M. Tshipa \\ University of Botswana, Private Bag 0022, Gaborone, Botswana \\ Tel.: +267-3554711 \\ Fax: $+267-3185097$ \\ E-mail: tshipam@ub.ac.bw \\ ORCID: 0000-0001-6894-7637
}


rates are found to be characterized by shallow minima in their variations with the inner radius, again, for a fixed outer radius. This feature is a consequence of a balance between two seemingly conflicting effects of the electron's confinement by the inner and outer walls of the hollow cylinder. First; increased confinement of the charge carriers generally leads to the enhancement of the rates. Second; the presence of a hole in a hollow cylinder leads to a significant suppression of the scattering rates. The intrasubband scattering rates also show a somewhat parabolic increase in their variations with the applied magnetic field; an increase which is more pronounced in a relatively thick hollow cylinder.

Keywords Electron-phonon interaction · Scattering rates · Hollow cylinder · Axial magnetic field

\section{Introduction}

The rapid advancement in fabrication technologies (Liang et al., 2019; Fu et al., 2020) as well as experimental design (Beenakker and van Houten, 1991; Kim and Olendski, 1996) has led to a great deal of renewed interest in the interactions of confined electrons with the various crystal excitations, especially in semiconductors (Peeters and De Boeck, 1999). The renewed interest is in part stimulated by potential device applications (Fu et al., 2020; Khalef et al., 2020; Beretta et al., 2020), which mainly rely on reduced phase space of the charge carriers (Sakaki, 1980). As such, the nature of other physical quantum mechanical quantities depends on the specific dynamics of the confined electrons, which in turn is largely determined by the overall potential of a given system. The overall potential of low-dimensional systems falls into two broad categories: a) Confinement of the charge carriers may be due to the intrinsic socalled electric potential of the fabricated nanostructures (Tonucci et al., 1992; Goldberger et al., 2003). This form of the potential depends on the specific topology or even the geometrical symmetry of the quantum structure (Santos Jr. et al., 2005; Filikhin et al., 2006). For example, the angular momentum of an electron is inherently quantized in integral units of Planck's constant in cylindrical nanosystems but not necessarily so in plano-wall quantum wells. b) Additional confinement of electrons may also be due to the vector potential, in general, of a spatially inhomogeneous applied magnetic field (Badran and Ulloa, 1999; Reijniers et al., 2001; Masale, 2004, 2008). The other factor which plays a crucial role in the specific character of the properties of the confined charge carriers is the orientation of an applied magnetic field relative to the interface of a heterojunction (Masale, 2004, 2008). In recent years, there has been a surge of interest in carbon nanotubes, which almost overshadows that of their semiconductor counterparts; very thin cylindrical shells. Apart from their comparatively high geometrical regularity, carbon nanotubes display a broad range of electronic, thermal and structural properties that change depending on their dimensions and chirality (Saeed and Khan, 2013). Indeed, it is envisaged that carbon nanotubes are formidable candidates for future nanodevice 
applications. Notwithstanding this greater potential of carbon nanotubes for future nanotechnologies, the physics of semiconductor nanostructures continues to attract attention of among researchers (Hien et al., 2017), and the other references therein. In part, this is because carbon nanotubes can indeed exhibit either metallic or semiconductor properties, as stated earlier, depending on their dimensions and chirality.

Scattering of electrons by phonons, particularly via polar LO modes, is one of the most important energy relaxation processes in low-dimensional systems (Alcalde and Weber, 2000; Bai et al., 2019; Quoc and Dinh, 2019). For example, they play a significant role in decoherence in quantum computing (Fotue et al., 2015; Khordad and Ghanbari, 2017; Tiotsop et al., 2018), in promoting stable hot carrier population in solar cells (Garg and Sellers, 2020) and in photoluminescence (Ding et al., 2017). Even though phonons can be responsible for the decoherence of qubits (Kornich et al., 2018), there is some indication that they may also play a positive role in the quantum information industry. Research has shown that robust coherence can be maintained in a double quantum dot system due to the presence of phonons (Purkayastha et al., 2020). Recently, phonons have been used to couple distant quantum systems, facilitating quantum entanglement of two qubits (Bienfait et al., 2019). This transfer of coherent phonons has also been observed experimentally between bismuth nanofilms and a glass substrate (He et al., 2020). Studies of longitudinal optical phonons in $G a A s / A l_{x} G a_{1-x} A s$ quantum core-shell wires have shown that phonons influence the mobility of electrons, and that mobility can be achieved by core-shell wires of thicker shells (Dat and Hai, 2019). Longitudinal optical phonons have also been shown to reduce binding impurity energies in spherical core/shell quantum dots (Talbi et al., 2021). Phonon modes are influenced by topology, and the Möbius topology has been shown to give rise to phonon band gaps (Nishiguchi and Wybourne, 2018). Phonon band gaps can be useful and have been utilized to block resonant decay of defect states, thereby protecting qubits (Rosen et al., 2019). There are various modes of the phonon spectrum in low-dimensional structures; among these; the bulk-like confined modes and the interface modes (Hai et al., 1993). Now, the total electron-phonon scattering rate; assumed to be the sum of the contributions of the above mentioned individual phonon modes; has been found to be more or less equal to the value obtained using the bulk phonon spectrum (Mori and Ando, 1989; Rücker and Molinari, 1992; Register, 1992). Understanding scattering rates is crucial since other quantities depend on them, from example, phonon-drag thermopower (Tsaousidou, 2019) and thermal conductivity (Feng and Ruan, 2018; Zhu et al., 2019; Dongre et al., 2020). There are now numerous well established experimental techniques for probing electronphonon interactions; including spectroscopic measurements (Mirlin and Perel, 1992; Tsen et al., 1998) and femtosecond time-resolved differential transmission (Schumacher et al., 1996).

The main aim of the investigations undertaken here is to evaluate the ground-state intrasubband electron-LO-phonon scattering rates as functions of axial applied magnetic field in a hollow cylinder. The scattering rates are 
evaluated assuming the bulk phonon spectrum and within the framework of the Fröhlich interaction Hamiltonian, rather in the spirit of Mori and Ando (Mori and Ando, 1989). As remarked earlier, new features of the quantum properties of the confine charge carriers emerge merely as a consequence of altering the topological symmetry of a quantum system (Masale, 2003). It is worth mentioning that the character of quantum processes involving electrons to a large extent depends on the specific nature of the electron's energy spectrum. As mentioned earlier, the specific character of the electron's spectrum itself depends on the overall confinement as well as the geometrical symmetry of the system considered. In this regard, of particular interest here, at least qualitatively, is how the topological evolution from a solid to a hollow cylinder influences the ground-state intrasubband scattering rates.

\section{Electron states}

The system studied here is a long hollow cylinder of inner and outer radii $R_{1}$ and $R_{2}$, respectively, immersed in a uniform axial applied magnetic field $\mathbf{B}=$ $(0,0, B)$. The two forms of the potential considered are the vector potential: $\mathbf{A}=\left(0, \frac{1}{2} B \rho, 0\right)$ of the uniform axial applied magnetic field, and the so-called electric potential; taken in the form:

$$
V(\rho)=\frac{1}{2} \mu \omega_{o}^{2}\left(\rho^{2}-R_{1}^{2}\right) \quad \text { for } \quad R_{1} \leq \rho \leq R_{2}
$$

and infinity elsewhere. The parameter $\omega_{o}$ is the angular frequency of a simple harmonic oscillator of effective mass $\mu$; assumed to be equal to that of an electron in a semiconducting material. This form of the electric potential resembles somewhat the true potential of a heterojunction formed between AlGaAs and GaAs (Schlesinger et al., 1983). In view of cylindrical symmetry, the electron's wave function corresponding to the $\ell$ th radial and $m$ th azimuthal quantum numbers is taken in the general form:

$$
\psi_{\ell m}=C_{N} \exp \left(i k_{z} z\right) \exp (i m \varphi) \chi(\rho) \quad m=0, \pm 1 \pm 2 \pm 3 \ldots,
$$

where $k_{z}$ is the electron's axial wavenumber and $C_{N}$ is the nomalization constant. The Schrödinger equation for this system is solvable in terms of the confluent hypergeometric function. In particular, $\chi$ is taken as a linear combination of the $M$ and $U$ confluent hypergeometric functions:

$$
\chi=\zeta^{|m| / 2} \exp (-\zeta / 2)[A M(a, b, \zeta)+B U(a, b, \zeta)]
$$

where $A$ and $B$ are constants, which are determined from the application of boundary conditions. The particular parameters $a$ and $b$ and the argument $\zeta$ of the confluent hypergeometric function are given as follows

$a=\frac{1}{2}+\frac{1}{2}|m|-\left[E_{\ell m}+\frac{1}{2} \mu \omega_{o}^{2} R_{1}^{2}-\frac{1}{2} m \hbar \omega_{c}\right] / \hbar \omega_{\tau}, \quad b=|m|+1 \quad$ and $\quad \zeta=\frac{\mu}{2 \hbar^{2}} \hbar \omega_{\tau} \rho^{2}$, 
where

$$
\omega_{\tau}=\sqrt{\omega_{c}^{2}+4 \omega_{o}^{2}}
$$

in which $\omega_{c}=e B / \mu$ and $a_{c}=(\hbar / e B)^{\frac{1}{2}}$ are the cyclotron parameters of the frequency and the radius of a particle of charge $e$, respectively. The application of the boundary condition of continuity of $\psi$ across the surfaces of the hollow cylinder: $\rho=R_{i} ; i=1$ or 2 ; leads to the following eigenvalue equation for the determination of the electron's subband energies

$$
U\left(a, b, \zeta_{R_{1}}\right) M\left(a, b, \zeta_{R_{2}}\right)-U\left(a, b, \zeta_{R_{2}}\right) M\left(a, b, \zeta_{R_{1}}\right)=0,
$$

where $\zeta_{R}$ is given by equation (4) for $\zeta$ but with the appropriate replacement: $\rho=R_{i}$.

Figure 1 shows the variations of some few lowest-order electron's confinement energy subband segments with the applied magnetic field in a hollow cylinder of radii $R_{1}=50 \AA$ and $R_{2}=300 \AA$. The other relevant parameters are $\hbar \omega_{o}=$ $8.6 \mathrm{meV}$ and the effective-mass of the electron, relevant to the system of GaAs, is taken to be roughly 0.067 times that of the free electron $\left(m_{e}\right)$. Finally, the different energy subbands, all for $\ell=1$, may be identified according to their increasing intercepts by their azimuthal quantum numbers as $m=0, \pm 1$ and \pm 2 . In particular, the dashed curves correspond to $m=-1$ and $m=-2$ for the lower and higher curves, respectively. Note the Zeeman splitting of the same non-zero $|m|$ subbands, which are otherwise doubly degenerate in the absence of an axial applied magnetic field. However, in contrast to the analogous results of a solid cylinder, the character of a single-electron energy spectrum of a hollow cylinder immersed in a parallel applied magnetic field is such that the $\{m=0, \ell=1\}$ energy subband is not always the ground state. As the magnetic field is increased, instead, there arises a sequence of the ground state energy subbands corresponding to the series; $\{m=0,-1,-2,-3 \ldots\}$ of the azimuthal quantum numbers (Masale et al., 1992; Masale, 2000). This essentially means that the energy separations between the $m \leq 0$ energy subbands initially decrease with the increasing magnetic field. The energy subbands characterized by the $m \geq 0$, however, increase monotonically with an increase of the axial applied magnetic field.

Figure 2 shows the variations of some few lowest-order $(\{\ell=1,|m| \leq 2)$ electron's confinement energy subbands with the inner radius of the hollow cylinder for $B=20 T$ and a fixed outer radius: $R_{2}=300 \AA$. The other relevant parameters are as for figure 1 . The various curves stack up, at say $R_{1}=70 \AA$, according to their increasing azimuthal quantum numbers ranging from -2 to $m=2$. As anticipated, the subband energies increase with the decrease of the thickness of the hollow cylinder, due to the increased 'compression' of the wavefunction. The intersubband energy separations, however, decrease with the decrease of the thickness of the cylindrical shell. This is because in a very thin hollow cylinder, confinement by the walls of the hollow cylinder is much stronger than that due to the axial applied magnetic field. This is to 


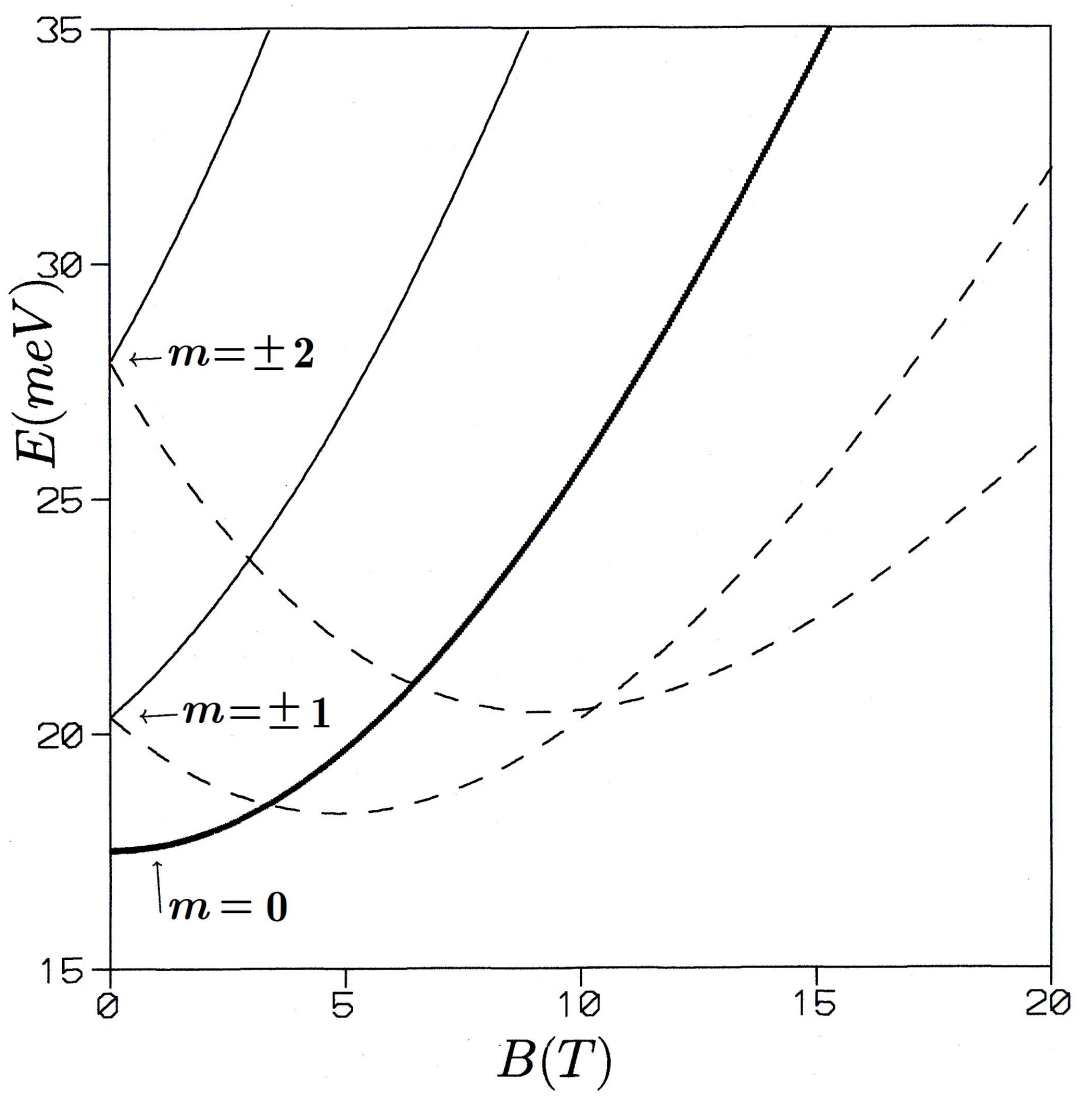

Fig. 1 Variations of some few lowest-order $(\{\ell=1,|m| \leq 2\})$ electron's confinement energy subbands with the applied magnetic field in a hollow cylinder of radii $R_{1}=50 \AA$ and $R_{2}=300 \AA$. The other relevant parameters are $\hbar \omega_{o}=8.6 \mathrm{meV}$ and $\mu=0.067 \mathrm{~m}_{e}$ where $m_{e}$ is the free-electron mass. The different subband energies are identified by their azimuthal quantum numbers indicated there; ranging from $m=-2$ up to $m=2$. In particular, the dashed curves correspond to negative azimuthal quantum numbers

say that in the regime of ultra thin cylindrical shells, the subband energies are somewhat insensitive to either the applied magnetic field or the electron's subband azimuthal quantum numbers. It should also be recalled that unlike in a solid cylinder, the boundary condition of continuity of the wavefunction at $R_{1}$ in a hollow cylinder drastically changes the phase of the $\{\ell=1, m=0\}$ electron's eigenfunction there. 


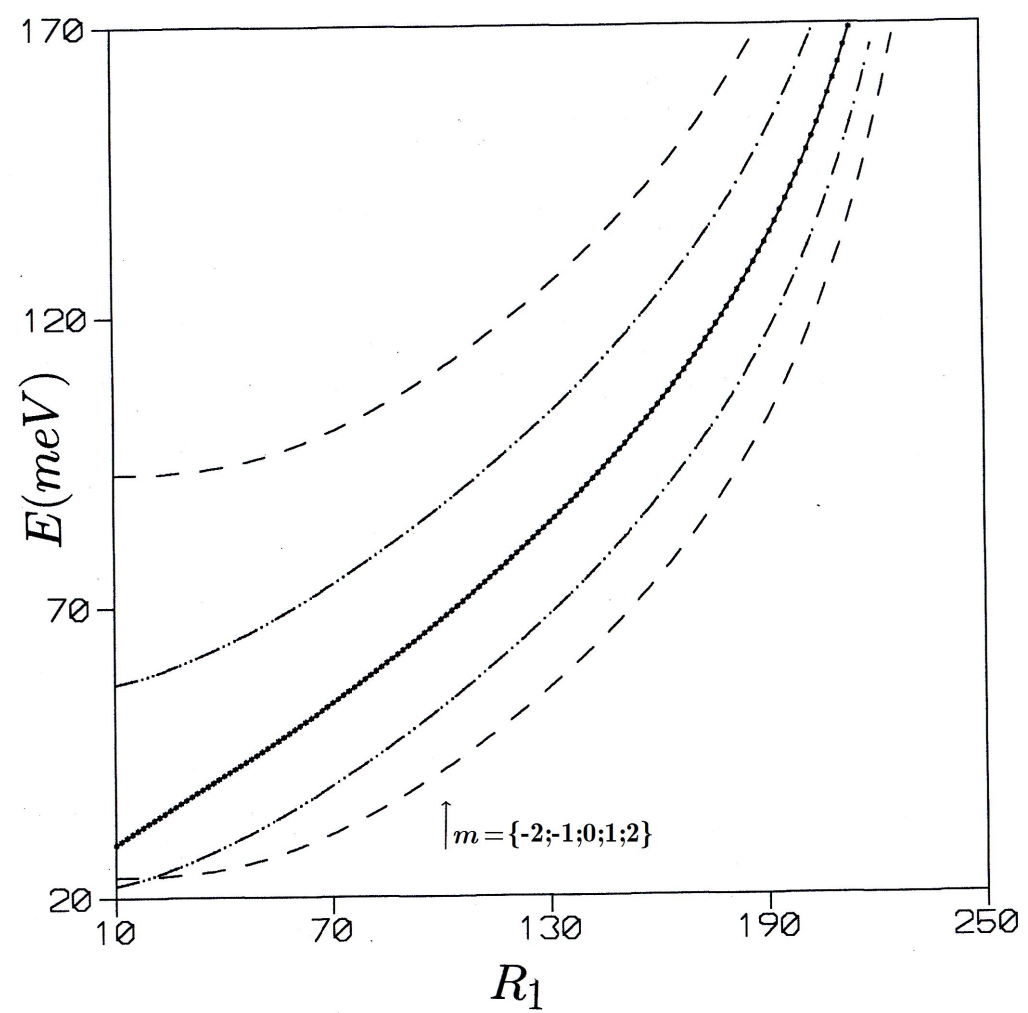

Fig. 2 Variations of some few lowest-order $(\{\ell=1,|m|<2)$ electron's confinement energy subband with the inner radius of the hollow cylinder for $B=20 T$ and a fixed outer radius: $R_{2}=300 \AA$. The other relevant parameters are as for figure 1 . The various curves stack up, at say $R_{1}=70 \AA$, according to their increasing azimuthal quantum numbers ranging from -2 to $m=2$, as indicated there

\section{Electron-LO-phonon scattering rates}

Rather briefly, lattice displacements in the optic longitudinal mode produce a macroscopic electric field, $\mathbf{E}=-\nabla \Phi$, which couples electrons to the lattice vibrations through the scalar potential $\Phi$. As mentioned earlier, the evaluations of the scattering rates here, are carried out employing the Fröhlich interaction Hamiltonian, $H_{\text {int }}=-e \Phi$, given by (Fröhlich, 1954):

$$
H_{i n t}=\sum_{q} C(q)\left[\mathbf{a}_{q} \exp (i q \cdot \mathcal{R})+\mathbf{a}_{q}^{\dagger} \exp (-i q \cdot \mathcal{R})\right]
$$


where $\mathbf{a}_{q}$ and $\mathbf{a}_{q}^{\dagger}$ are the phonon creation and annihilation operators, respectively, $\mathcal{R}$ is the position vector and $C(q)$ is a coupling factor, which for LOphonons, is given by

$$
C(q)=\frac{i}{q}\left[\left(e^{2} \hbar \omega_{L} / 2 \varepsilon_{o} V_{C}\right)\left(\varepsilon_{\infty}^{-1}-\varepsilon_{s}^{-1}\right)\right]^{1 / 2},
$$

in which $\varepsilon_{o}$ is the permittivity of free space. The other parameters, relevant to the system of GaAs, are the high- and low-frequency dielectric constants $\varepsilon_{\infty}=$ 10.9 and $\varepsilon_{s}=13.1$, respectively. Finally, $\omega_{L}$ is the LO-phonon zone-center frequency, corresponding to the LO-phonon energy quantum: $\hbar \omega_{L}=36.6 \mathrm{meV}$. The two basic processes described by this interaction Hamiltonian are annihilation or creation of a bulk phonon of wave vector $\mathbf{q}$, with axial $\left(q_{z}\right)$ and off-axial $\left(q_{r}\right)$ components such that $q^{2}=q_{r}^{2}+q_{z}^{2}$. Either of these processes is accompanied by a change of state of the electron, from the initial $\left|\ell, m, k_{z}\right\rangle$ to the final $\left|\ell^{\prime}, m^{\prime}, k_{z}^{\prime}\right\rangle$. For these processes occurring in quasi-one-dimensional systems, overall conservation of the axial momentum is prescribed by $k_{z}^{\prime}=k_{z}+q_{z}$ and $k_{z}^{\prime}=k_{z}-q_{z}$ for absorption and for emission of a phonon, respectively. The system considered here is an addition to the family of systems with cylindrical symmetry for the closely related investigations on electron phonon interactions (Masale, 2003; Masale and Constantinou, 1993; Leão et al., 1993) and are included here only for completeness. In particular, the emission $\Gamma^{e m}$ scattering rates are given by

$$
\Gamma^{e m}=\frac{2 \Gamma_{o}\left[n\left(\omega_{L}\right)+1\right]}{\sqrt{\varepsilon_{i}-(1-\Delta \varepsilon)}} \int_{0}^{\infty} y\left|I_{\ell m \ell^{\prime} m^{\prime}}(y)\right|^{2}\left[\frac{1}{y^{2}+Q_{+}^{2}}+\frac{1}{y^{2}+Q_{-}^{2}}\right] d y,
$$

where $n\left(\omega_{L}\right)=\left[\exp \left(\hbar \omega_{L} / k_{B} T\right)-1\right]^{-1}$ is the usual temperature-dependent Bose-Einstein distribution function, in which $k_{B}$ is the Boltzmann constant. The interaction integral is given by

$$
I_{\ell m \ell^{\prime} m^{\prime}}(y)=\int_{R_{1} / R_{2}}^{1} x J_{\left|m-m^{\prime}\right|}(y x) \psi_{\ell m}(x) \psi_{\ell^{\prime} m^{\prime}}^{*}(x) d x,
$$

where the dimensionless radial distance and the reduced radial phonon wavenumber are given by $x=\rho / R_{2}$ and $y=q_{\rho} R_{2}$, respectively. The other variables appearing in equation (9) are the scaled energies:

$$
\varepsilon_{i}=E_{k} / \hbar \omega_{L} \text { and } \Delta \varepsilon=\left[\left|E_{\ell m}-E_{\ell^{\prime} m^{\prime}}\right|\right] / \hbar \omega_{L}
$$

as well as the scaled phonon wavenumbers

$$
Q_{ \pm}=\sqrt{\left(2 \mu \omega_{L} R_{2}^{2} / \hbar\right)}\left(\sqrt{\varepsilon_{i}} \mp \sqrt{\varepsilon_{i}-1+\Delta \varepsilon}\right),
$$

wherein the plus $(+)$ and minus (-) signs refer to forward and backward scattering of phonons, respectively. Finally, the characteristic scattering rate $\Gamma_{o}$ 
(typically $8.7 \times 10^{12} s^{-1}$, for GaAs) is defined in terms of the Fröhlich coupling constant, $\alpha$, according to:

$$
\Gamma_{o}=2 \alpha \omega_{L}=e^{2} /\left(4 \pi \varepsilon_{o} \hbar\right)\left[\varepsilon_{\infty}^{-1}-\varepsilon_{s}^{-1}\right]\left[2 \mu \omega_{L} / \hbar\right]^{1 / 2} .
$$

It is worth mentioning that intersubband scattering can be crucial in determining the lifetimes of the electronic states. However, the main results of these investigations; the scattering rates; are illustrated only for transitions within the $\{\ell=1, m=0\}$ electron's energy subband. As such, in most cases of practical interest, these are usually the strongest transitions. For the system considered here, in general, the intersubband energy separations are less than the LO-phonon energy quantum except for high values of the applied magnetic fields and large differences of the subband quantum numbers (Masale et al., 1992). Apart from these somewhat extreme cases therefore, intersubband emission scattering of an LO-phonon in general would be forbidden. In loose terms, the electron simply does not have enough energy to emit an LOphonon whenever the electronic intersubband energy separations are less than the phonon energy quantum. Now, the variations of the absorption scattering rates with the applied magnetic field, although much lower, have been found to be the same as those of the corresponding emission rates. The numerical results for absorption rates are thence not shown here, for brevity.

Figure 3 shows the variations of the room-temperature $(T \sim 300 K)\{\ell=$ $1, m=0\}$ intrasubband reduced emission scattering rates $\left(\Gamma^{e m} / \Gamma_{o}\right)$ with the inner radius $\left(R_{1}\right)$ for some few values of the applied magnetic field. The key relevant parameters are as for figure 1 , in particular, $R_{2}=300 \AA$. The additional relevant parameter is the so-called axial electron's kinetic energy: $\epsilon_{i}=1.001$, taken to be just in excess of the LO phonon energy quantum. The curves stackup according to their corresponding values of the applied magnetic field used: $B \sim 0 T$ for the lowest curve, then increasing in steps of $\Delta B=5 T$ up to $20 T$ for the highest curve. Note that the intrasubband scattering rates are higher for larger values of the axial applied magnetic field particularly in relatively thick hollow cylinders. This is to say that the scattering rates become more enhanced with increased confinement of the electron by the applied magnetic field. It is also seen that the intrasubband scattering rates initially decrease rapidly and are characterized by show shallow minima in their variations with the inner radius. Now, the nature of these scattering rates is determined by a balance between two conflicting factors resulting from increasing $R_{1}$, for a fixed outer radius. First, these become enhanced with increased confinement by the walls of the hollow cylinder. Second, the scattering rates become increasingly suppressed because of the 'dilation' of the forbidden region $\left(\rho \leq R_{1}\right)$ for the motion of the electrons (Masale, 2003). Note that the evolution from a solid to a thin shell (increasing $R_{1}$ ) may be regarded as perturbing the quasione-dimensionality of the electronic density of states of a solid cylinder. This is the effect that is thought to lead to the quenching of the coherence of the electron-phonon Fröhlich coupling (Fröhlich, 1954). 


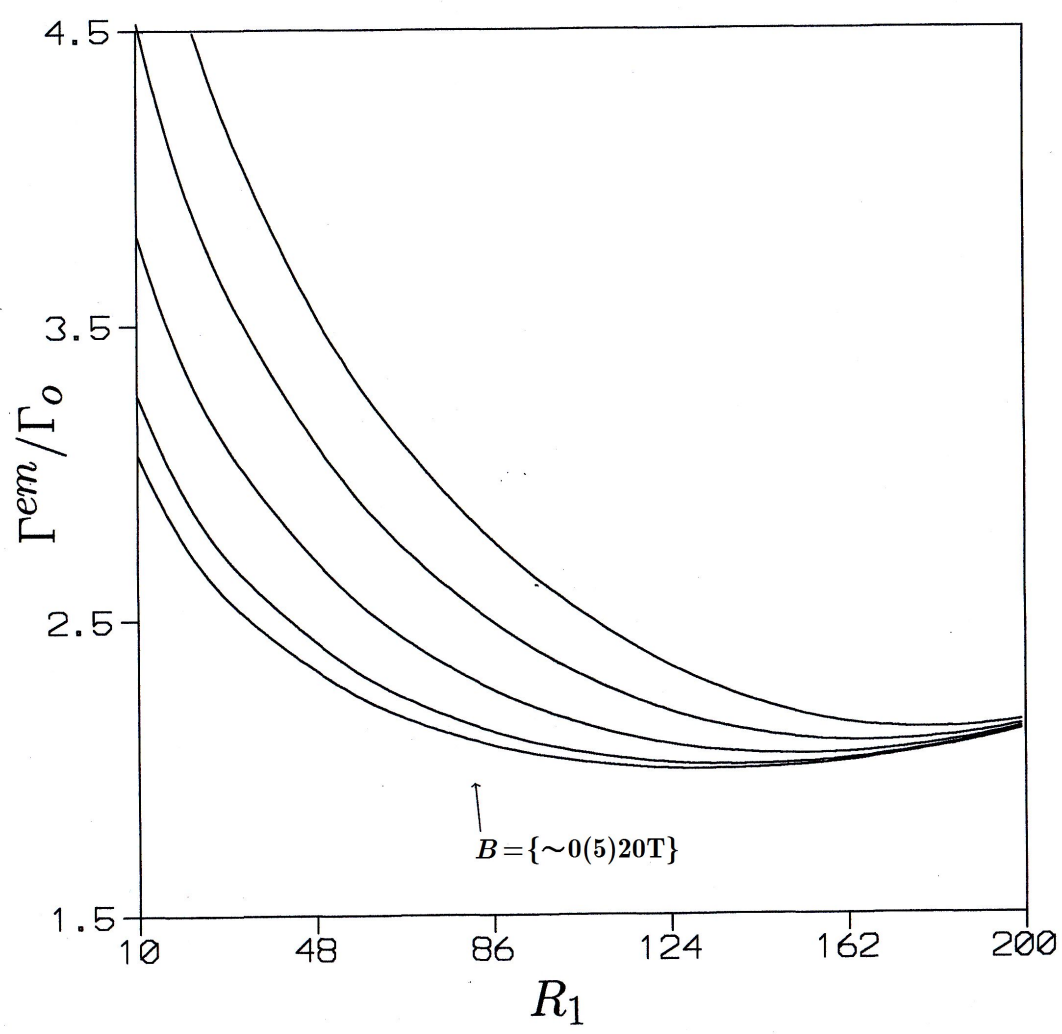

Fig. 3 The room-temperature $(T \sim 300 K)\{\ell=1, m=0\}$ intrasubband reduced emission scattering rates versus the inner radius for some few values of the applied magnetic field shown there. The curves stack-up according to the increasing values of the magnetic: $B \sim 0 T$ for the lowest, then increasing in steps of $5 T$ up to $20 T$ for the highest curves, respectively. The key relevant parameters are as for figure 1 except $\epsilon_{i}=1.001$

Figure 4 depicts the variations of $\Gamma^{e m} / \Gamma_{o}$ with $B$ for some few values of the thickness of the hollow cylinder. The other relevant parameters, except for $R_{1}$, are as for figure 3 . For ease of presentation of the results, figure $4 \mathrm{a}$ and figure $4 \mathrm{~b}$ may be regarded as depicting the results for the regime relatively thick and thin hollow cylinders, respectively. The curves in figure 4a stack-up according to the increasing thickness of the hollow cylinder, essentially varying $R_{1}$, for a fixed outer radius: $R_{2}=300 \AA$. To be more precise, in figure $4 \mathrm{a}$, the highest curve corresponds to $R_{1}=50 \AA$, the inner radius then increased in steps of $10 \AA$ up to $R_{1}=140 \AA$ for the lowest curve. Following the reverse trend of the stacking-up of the various curves, in figure $4 \mathrm{~b}$, the inner radius ranges from $R_{1}=150 \AA$ for the lowest curve up to $R_{1}=200 \AA$ for the highest curve. Again, it is worth commenting that the intrasubband scattering rates are generally somewhat insensitive to the electron's subband azimuthal 


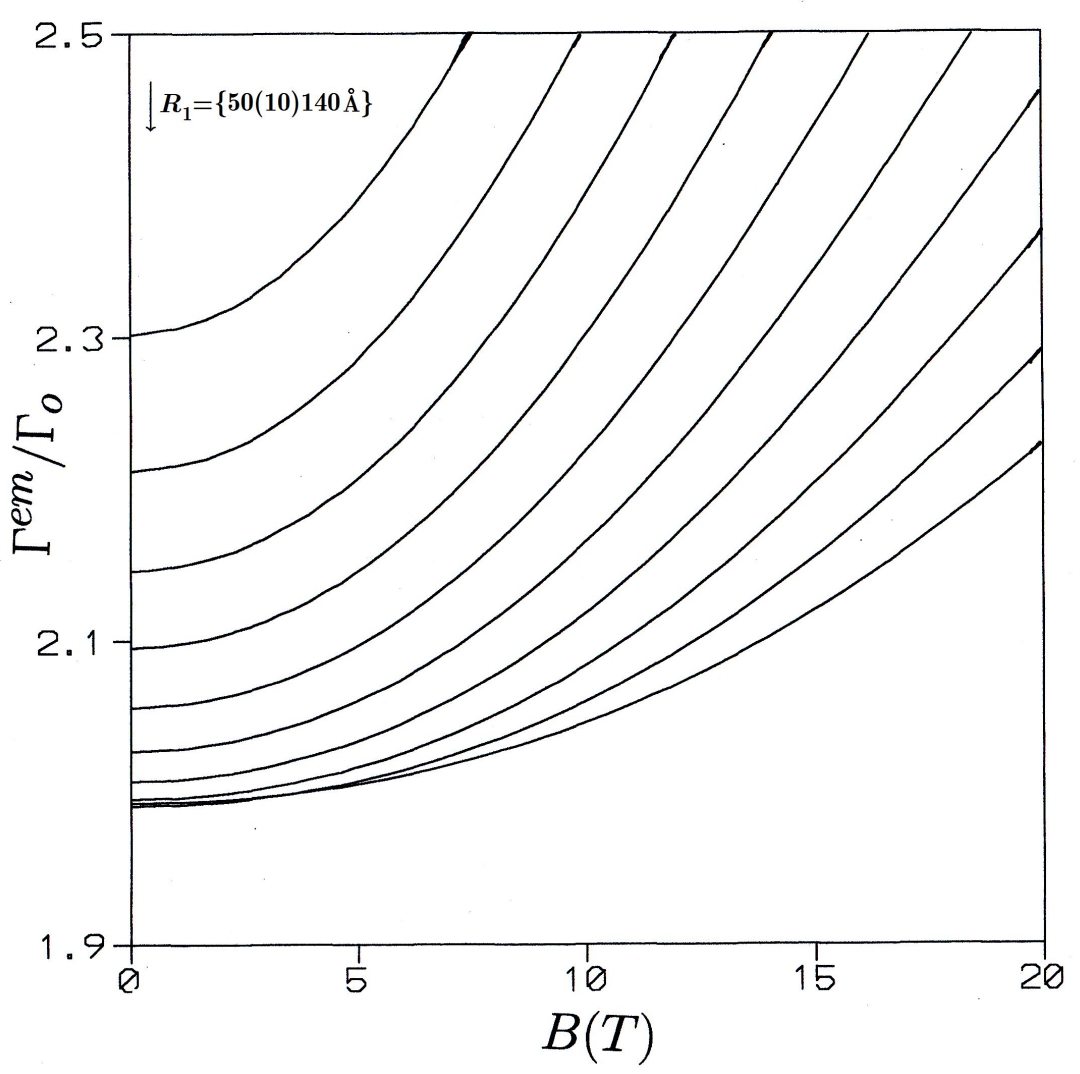

Fig. 4a Variations of $\Gamma^{e m} / \Gamma_{o}$ with $B$ for some few values of the thickness of the hollow cylinder. The other relevant parameters, except for $R_{1}$, are as for figure 3 . The curves stackup according to the increasing thickness of the hollow cylinder such that $R_{1}=140 \AA$ for the lowest curve, then decreasing in steps of $10 \AA$ down to $R_{1}=50 \AA$ for the highest curve

quantum numbers. In particular, unlike the Zeeman splitting of the $\pm m$ electron's energy subbands by the axial applied magnetic field, the corresponding results for the intrasubband scattering rates are still doubly degenerate. In other words, despite the difference in the corresponding electron's energies $\Delta E_{\ell m}=E_{\ell|m|}-E_{\ell-|m|}=\hbar \omega_{c}$, the relevant parameters $a$ and $b$ of the radial eigenfunctions of any such pair of azimuthal quantum numbers are exactly the same. In general, the intrasubband scattering rates increase with the increase of the axial applied magnetic field, particularly in the regime of relatively thick hollow cylinders. This feature is confirmed by figure $4 \mathrm{~b}$ wherein as the applied magnetic field is increased, the scattering rates for smaller $R_{1}$ rise above those corresponding to a larger inner radius. This behaviour is reminiscent of the $m \leq 0$ energy subbands taking turns at becoming the ground state as the axial applied magnetic field is increased, although here the azimuthal quan- 


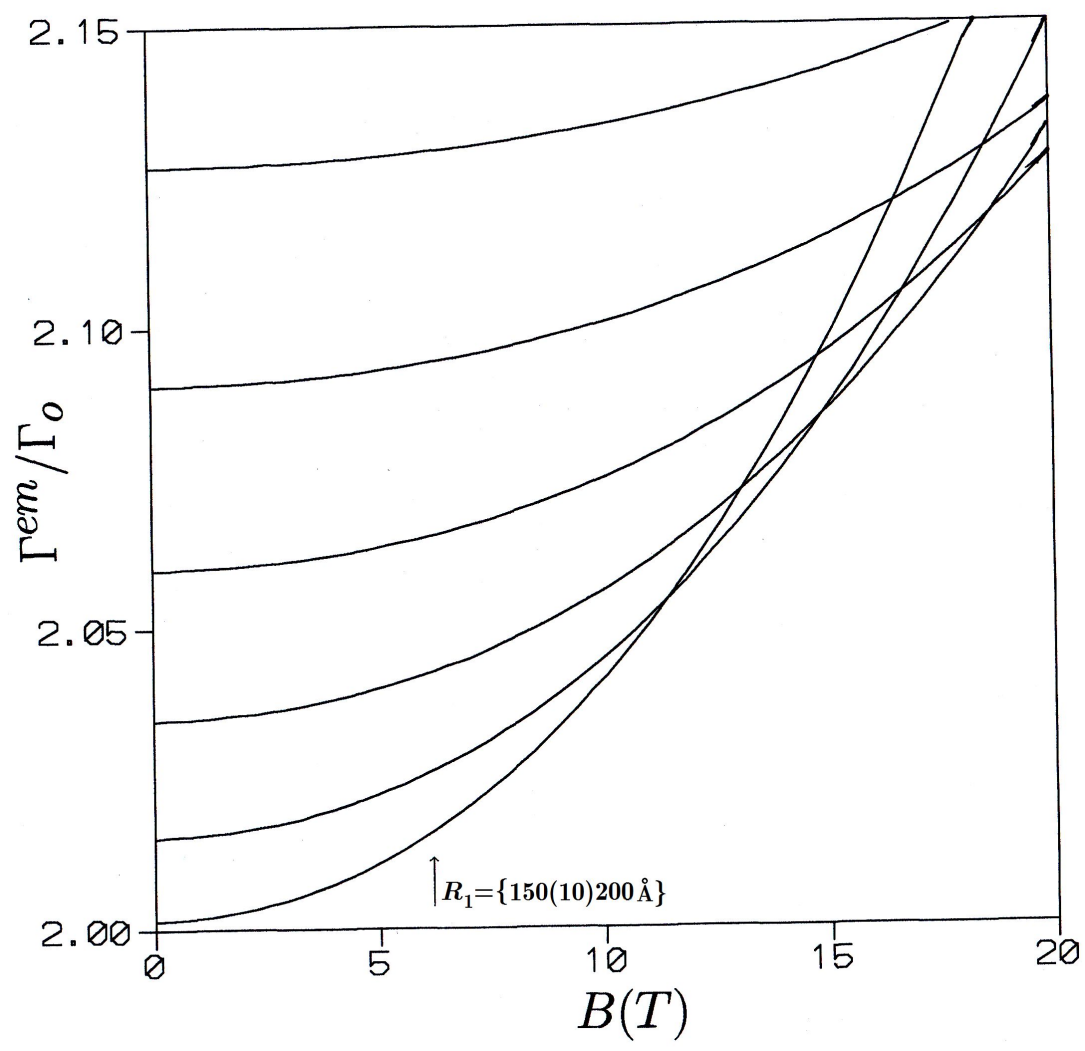

Fig. 4b Same caption as for figure 4a except for the larger values of the inner radius used Here, the curves stack-up according to the decreasing thickness of the hollow cylinder such that $R_{1}=150 \AA$ for the lowest curve, then increasing in steps of $10 \AA$ up to $R_{1}=200 \AA$, for the highest curve

tum number remains the same. As mentioned earlier, this behaviour of the scattering rates is essentially a signature of the presence of an inner forbidden region $\left(\rho<R_{1}\right)$ for the motion of the charge carriers under the influence an axial applied magnetic field. In other words, it is a consequence of a topological evolution of a solid into a hollow cylinder.

\section{Conclusions}

Electron-LO-phonon scattering rates of a hollow cylinder were calculated as functions of the inner radius as well as the axial applied magnetic field, assuming bulk LO-phonon modes. Now, the nature of quantum processes is largely dependent on the dispersion of the excitations involved. To this end, a resume was given of the energy spectrum of an electron confined in a hollow cylinder 
within the effective-mass approximation. The nature of this Zeeman splitting in a hollow cylinder is such that the $\{\ell=1, m \leq 0\}$ electron's energy subbands take turns at being the ground state, as the magnetic field is increased; following the sequence $\{m=0,-1,-2 \ldots\}$ of the azimuthal quantum numbers. The energy separations between the various electron's energy subbands of a hollow cylinder, by and large, were found to be less than the LO phonon energy quantum except for exceptionally high magnetic fields. The discussions of the results were thence focused on the lowest-order $\{\ell=1, m=0\}$ intrasubband scattering rates. These were deemed to be the most probable and in a sense representative of the ground-state intrasubband scattering rates. The lowestorder intrasubband scattering rates were found to increase with the increase of the axial applied magnetic field; notably for relatively thick hollow cylinders. The enhancement of the scattering rates is attributable to the increased confinement of the charge carriers due to the increase of the parallel applied magnetic field. The lowest-order scattering rates were also found to decrease with the increase of the inner radius but for a fixed outer radius, and for some few values of the applied magnetic field. An interesting feature is that the scattering rates are much enhanced for large values of the magnetic field and relatively thick hollow cylinders. Furthermore, in a very thin hollow cylinder, curves of the scattering rates corresponding to the different values of the magnetic field tend to merge. This effect signifies much stronger confinement of the electrons by the walls of the hollow cylinder than by the magnetic field. In general, the intrasubband scattering rates were found to possess shallow minima in their variations with the inner radius; for a fixed outer radius. These minima arise from a balance between the following two factors related to the topological evolution from a hollow to a solid cylinder. First, the enhancement of the scattering rates due to increased confinement of the walls of the hollow cylinder upon decreasing its thickness. Second, the quenching of the scattering rates arising from an expansion of the inner forbidden region for the motion of the charge carriers. As such, an increase of the above mentioned region may be regarded as breaking the quasi-one-dimensionality of electronic density of states; as would be the case in a solid cylinder. As a final comment, the results presented here give at least a semi-qualitative picture of the nature of the intrasubband electron-LO-phonon transitions in cylindrical systems. The character of these transitions should provide information required in the engineering of potential application of nanoscale devices.

\section{Declarations}

Funding: Not applicable.

Conflict of Interest: The authors declare that they have no conflict of interest. Availability of data and material: Not applicable.

Code availability: Not applicable. 


\section{References}

Alcalde, A. and Weber, G. (2000). Scattering rates due to electron-phonon interaction in cds1-xsex quantum dots. Semicond. Sci. Technol., 15:10821086.

Badran, E. and Ulloa, S. (1999). Frequency-dependent magnetotransport and particle dynamics in magnetic modulation systems. Phys. Rev. B, 59:2824.

Bai, X.-F., Zhao, Y.-W., Yin, H.-W., and Eerdunchaolu (2019). Influence of the electromagnetic field on the transition of a two-level system in a gaussian confinement potential with lo phonon and of thickness effects. Opt. Quant. Electron., 51:114.

Beenakker, C. and van Houten, H. andStaring, A. (1991). Influence of coulomb repulsion on the aharonov-bohm effect in a quantum dot. Phys. Rev. B, 44:1657.

Beretta, S., Bosi, M., Seravalli, L., and Musayeva, N.and Ferrari, C. (2020). Orientation of germanium nanowires on germanium and silicon substrates for nanodevices. Mater. Today-Proc., 20:30-36.

Bienfait, A., Satzinger, K. J., Zhong, Y. P., Chang, H.-S., Chou, M.-H., Conner, C. R., Dumur, E., Grebel, J., Peairs, G. A., Povey, R. G., and Cleland, A. N. (2019). Phonon-mediated quantum state transfer and remote qubit entanglement. Science, 364:368-371.

Dat, N. N. and Hai, L. T. (2019). Lo-phonon-limited electron mobility in a core-shell polar semiconductor quantum wire. Brazilian Journal of Physics, 49:628-635.

Ding, Q., Zhang, X., Li, L., Lou, X., Xu, J., Zhou, P., and Yan, M. (2017). Temperature dependent photoluminescence of composition tunable znxaginse quantum dots and temperature sensor application. Opt. Express, 25(16):19065-19076.

Dongre, B., Carrete, J., Wen, S., Ma, J., Li, W., Mingo, N., and Madsen, G. K. H. (2020). Combined treatment of phonon scattering by electrons and point defects explains the thermal conductivity reduction in highly-doped si. J. Mater. Chem. A, 8:1273.

Feng, T. and Ruan, X. (2018). Four-phonon scattering reduces intrinsic thermal conductivity of graphene and the contributions from flexural phonons. Phys. Rev. B, 97:045202.

Filikhin, I., V.M., S., and Vlahovic, B. (2006). Electron spectral properties of the inas/gaas quantum ring. Physica E, 33:349-354.

Fotue, A. J., Kenfack, S. C., Issofa, N., Tiotsop, M., Djemmo, M. P. T., Wirngo, A. V., Fotsin, H., and Fai, L. C. (2015). Decoherence of polaron in asymmetric quantum dot qubit under an electromagnetic field. American Journal of Modern Physics, 4(3):138-148.

Fröhlich, H. (1954). Electrons in lattice fields. Adv. Phys., 3:325.

Fu, X., Peng, F., Lee, J., Yang, Q., Zhang, F., Xiong, M., Kong, G., Meng, H.-M., Ke, G., and Zhang, X.-B. (2020). Aptamer-functionalized dna nanostructures for biological applications. Top. Curr. Chem., 378:21 (1-43. 
Garg, J. and Sellers, I. R. (2020). Phonon linewidths in inas/alsb superlattices derived from first-principles - application towards quantum well hot carrier solar cells. Semicond. Sci. Technol., 35:044001.

Goldberger, J., He, R., Zhang, Y., SLee, ., Yan, H., Choi, H.-J., and Yang, P. (2003). Single-crystal gallium nitride nanotubes. Nature, 422:599-602.

Hai, G., Peeters, F., and Devreese, J. (1993). Electron optical-phonon coupling in gaas $/ \mathrm{Pl}_{x} g a_{1-x}$ as quantum wells due to interface, slab, and half-space modes. Phys. Rev. B, 48:4666.

He, F., Walker, E. S., Zhou, Y., Muschinske, S. E., Bank, S. R., and Wang, Y. (2020). Quantum confinement of coherent acoustic phonons in transferred single-crystalline bismuth nanofilms. Appl. Phys. Lett., 116:263101.

Hien, N. D., Dinh, L., and Phong, T. C. (2017). Influence of phonon confinement on optically detected elecctrophonon resonance linewidth in parabolic quantum wires. Hue University Journal of Science: Natural Science, $126(1 \mathrm{~B}): 5-12$.

Khalef, W. K., Aljubouri, A. A., and Faisal, A. D. (2020). Photo detector fabrication based zno nanostructure on silicon substrate. Opt. Quant. Electron., $52: 334$

Khordad, R. and Ghanbari, A. (2017)). Effect of phonons on optical properties of rbcl quantum pseudodot qubits. Opt. Quant. Electron., 49:76.

Kim, C. and Olendski, O. . (1996). Landau levels and persistent currents in nonuniform magnetic fields. Phys. Rev. B, 53:12917.

Kornich, V., Vavilov, M. G., Frisen, M., and Coppersmith, S. N. (2018). Phonon-induced decoherence of a charge quadrupole qubit. New J. Phys., 20:103048.

Leão, S., Hipólito, O., and Peeters, F. (1993). Inter and intrasubband transitions via lo phonons in quantum wires. Superlattices Microstruct., 13:37.

Liang, X., Dong, R., and Ho, J. C. (2019). Self-assembly of colloidal spheres toward fabrication of hierarchical and periodic nanostructures for technological applications. Adv. Mater. Technol., 4:1800541 (1-9).

Masale, M. (2000). Oscillator strengths for optical transitions near a cylindrical cavity. Physica B, 291(3-4):256-265.

Masale, M. (2003). Dependence of the electron-lo-phonon intrasubband scattering rates near a cylindrical cavity on the axial applied magnetic field. Semicond. Sci. Technol., (7):661-665.

Masale, M. (2004). Single-electron states near a current-carrying core. Physica $B, 344: 284-291$.

Masale, M. (2008). Electron-lo-phonon scattering near a current-carrying core. Superlattices Microstruct., 43:269-277.

Masale, M. and Constantinou, N. (1993). Electron-lo-phonon scattering rates in a cylindrical quantum wire with an axial magnetic field: Analytic results. Phys. Rev B, 48:11128.

Masale, M., Constantinou, N., and Tilley, D. (1992). Single-electron energy subbands of a hollow cylinder in an axial magnetic field. Phys. Rev B, $46: 15432$. 
Mirlin, D. and Perel, V. (1992). Spectroscopy of Nonequilibrium Electrons and Phonons. North-Holland, Amsterdam.

Mori, N. and Ando, T. (1989). Electron-optical-phonon interaction in single and double heterostructures. Phys. Rev B, 40:6175.

Nishiguchi, N. and Wybourne, M. N. (2018). Phonon modes in a möbius band. J. Phys. Commun., 2:085002.

Peeters, F. and De Boeck, J. (1999). Handbook of Nanostructured Materials and Technology. New York: Academic.

Purkayastha, A., Guarnieri, G., Mitchson, M. T., Filip, R., and Goold, J. (2020). Tunable phonon-induced steady-state coherence in a doublequantum-dot charge qubit. Quantum Information, 6:27.

Quoc, K. D. and Dinh, H. N. (2019). Influence of confined phonon for the different models in gaas quantum wells on the optically detected electrophonon resonance linewidth. Opt. Quant. Electron., 51:116.

Register, L. (1992). Microscopic basis for a sum rule for polar-optical-phonon scattering of carriers in heterostructures. Phys. Rev B, 45:8756.

Reijniers, J., Peeters, F., and Matulis, A. (2001). Electron scattering on circular symmetric magnetic profiles in a two-dimensional electron gas. Phys. Rev. B, 64:245314.

Rosen, Y. J., Horsley, M. A., Harrison, S. E., Holland, E. T., Chang, A. S., Bond, T., and DuBois, J. L. (2019). Protecting superconducting qubits from phonon mediated decay. Appl. Phys. Lett., 114:202601.

Rücker, H. and Molinari, E. andLugli, P. (1992). Microscopic calculation of the electron-phonon interaction in quantum wells. Phys. Rev. B, 45:6747.

Saeed, K. and Khan, I. (2013). Carbon nanotubes-properties and applications: a review. Carbon Lett., 14(3):131-144.

Sakaki, H. (1980). Scattering suppression and high-mobility effect of sizequantized electrons in ultrafine semiconductor wire structures. Jpn. J. Appl. Phys., 19:L735-L738.

Santos Jr., D., Qu, F., Alcalde, A. M., and Morais, P. (2005). Influence of the quantum dot shape on the determination of the electronic structure and electron decoherence. Physica E, 26:331.

Schlesinger, Z., J.M.C., H., and Allen Jr., S. J. (1983). Subband-landau-level coupling in a two-dimensional electron gas. Phys. Rev. Lett., 50:2098.

Schumacher, K., Collings, D., Phillips, R., Ritchie, D., Weber, G., Schulman, J., and Ploog, K. (1996). Inter- and intrasubband relaxation times in gaas $-a l_{0.35} g a_{0.65}$ as quantum wells measured by femtosecond time-resolved differential transmission. Semicond. Sci. Technol., 11:1173.

Talbi, A., El Haouari, M., Nouneh, K., Pérez, L. M., Tiutiunnyk, A., Laroze, D., Courel, M., Mora-Ramos, M. E., and Feddi, E. (2021). Lo-phonons and dielectric polarization effects on the electronic properties of doped gan/inn spherical core/shell quantum dots in a nonparabolic band model. Applied Physics A, 127:30.

Tiotsop, M., Fotue, A. J., Fotsin, H. B., and Fai, L. C. (2018). Application of entropies to the study of the decoherence of magnetopolaron in 0-d nanosystem. Optical and Quantum Electronics, 50:365. 
Tonucci, R., Justus, B., Campillo, A., and Ford, C. (1992). Nanochannel array glass. Science, 258:783.

Tsaousidou, M. (2019). Enhanced phonon-drag thermopower of ballistic semiconducting single-walled carbon nanotubes near the second subband edge. Phys. Status Solidi B, 2019:1800757.

Tsen, K., Ferry, D., Salvador, A., and Morkoc, H. (1998). Picosecond raman studies of the electron-phonon interactions in $a l_{x} g a_{1-x} a s$ : Nonmonotonic dependence upon the alloy composition. Phys. Rev. Lett., 80:4807.

Zhu, L., Li, W., and Ding, F. (2019). Giant thermal conductivity in diamane and the influence of horizontal reflection symmetry on phonon scattering. Nanoscale, , ,, 11:4248. 